\title{
The star formation process in the Magellanic Clouds
}

\author{
J. M. Oliveira \\ Lennard-Jones Laboratories, School of Physical \& Geographical Sciences, Keele University, \\ Staffordshire ST5 5BG, UK \\ email: joana@astro.keele.ac.uk
}

\begin{abstract}
The Magellanic Clouds offer unique opportunities to study star formation both on the global scales of an interacting system of gas-rich galaxies, as well as on the scales of individual star-forming clouds. The interstellar media of the Small and Large Magellanic Clouds and their connecting bridge, span a range in (low) metallicities and gas density. This allows us to study star formation near the critical density and gain an understanding of how tidal dwarfs might form; the low metallicity of the SMC in particular is typical of galaxies during the early phases of their assembly, and studies of star formation in the SMC provide a stepping stone to understand star formation at high redshift where these processes can not be directly observed. In this review, I introduce the different environments encountered in the Magellanic System and compare these with the Schmidt-Kennicutt law and the predicted efficiencies of various chemophysical processes. I then concentrate on three aspects that are of particular importance: the chemistry of the embedded stages of star formation, the Initial Mass Function, and feedback effects from massive stars and its ability to trigger further star formation.
\end{abstract}

Keywords. astrochemistry, stars: formation, stars: luminosity function, mass function, ISM: clouds, H II regions, ISM: molecules, galaxies: evolution, Magellanic Clouds, galaxies: stellar content

\section{Introduction}

The Magellanic Clouds (MCs) are our closest gas-rich galaxy neighbours. New instrumental advances mean we can now study their resolved stellar populations in great detail. At the same time, in the MCs we can study these populations from outside the galaxies themselves, allowing us a unique view of these populations and how they relate to the gas and dust distributions and the galaxies' structure.

The formation of stars in the early Universe took place in a metal-poor environment, however most of what is currently known about the star formation process is derived from observations in the Milky Way. A great advantage of the MCs is that their Interstellar Media (ISM) are characterised by metallicity significantly lower than that of the Milky Way. Thus the MCs are ideal templates to test whether metallicity significantly influences star formation and thus are unique probes for the environmental conditions more typical of the high-redshift Universe.

Only since relatively recently are we able to study the details of the star formation in the MCs. Firstly, I describe the star formation environment in the Magellanic Clouds. I will then concentrate on three particular facets of the star formation process. I will start by discussing the effects of a lower metal content on the chemistry in molecular clouds. I will then discuss the stellar Initial Mass Function (IMF), as well as massive star feedback and triggered star formation at low metallicity. 


\section{The star formation environment in the MCs}

In the Milky Way star formation is clearly dominated by the spiral arms (see review by Elmegreen 2009). In galaxies with weak or no spiral arms, star formation seems to occur throughout their disks, probably resulting from local gravitational instabilities. This is the case of the MCs, that exhibit no clear spiral arms but are still observed to be forming stars at present $\dagger$. On the other hand, the MCs are interacting gas-rich galaxies that also interact with the Milky Way. Thus tidal and/or hydrodynamical effects might have an important role in stimulating and regulating star formation in these galaxies. The properties and location of the young stellar populations identified in the SMC tail (the part of the Magellanic Bridge closest to the SMC) suggest that these stars formed within a body of gas that had already been pulled out of the SMC, probably by tidal forces (Harris 2007). Whether these tides were also responsible for triggering the star formation is not clear. It has been proposed that the formation of the giant complex 30 Doradus could be associated with the last Magellanic collision, about 0.2 Gyr ago (Bekki \& Chiba 2007), which may have induced a gaseous spiral arm distortion lasting more than an orbital period. The alternative explanation of ram-pressure induced star formation as a result of the LMC ploughing through the hot Galactic halo (de Boer et al. 1998) has recently gained support again, explaining also some features of the Magellanic Stream (Nidever, Majewski \& Burton 2008).

The star formation efficiency is generally observed to be correlated with the local gas density. This Schmidt law (Schmidt 1959) — and its generalization to include starbursts by Kennicutt (1998) — is usually expressed in terms of the projected star formation rate and gas column density. It is observed that the efficiency of star formation is lower when the gas density is higher, and there appears to be a lower threshold to the gas density that can support star formation (Kennicutt 1998). Typical gas densities for distinct components in the Magellanic System may be estimated from the H I data presented in Nidever et al. (2008, see their Fig. 9), where the H I column density was transformed into total gas density assuming that the atomic and molecular gas fractions are equal (see Fig. 4 in Kennicutt 1998), a common average distance of $60 \mathrm{kpc}$, and ignoring projection effects. These are listed in Table 1 and compared with the Schmidt-Kennicutt law in Fig. 1. Note that the molecular fraction of gas in the Bridge/Stream is likely to be smaller.

Table 1. Relevant properties of different regions in the Magellanic System. Typical gas densities are estimated from Nidever et al. (2008).

\begin{tabular}{lcccl}
\hline Region & $Z / \mathrm{Z}_{\odot}$ & $\begin{array}{c}\mathrm{N}(\mathrm{H} \mathrm{I}) \\
\text { atoms cm }\end{array}$ & $\begin{array}{c}\sum(\text { gas }) \\
\mathrm{M}_{\odot} \mathrm{pc}^{-2}\end{array}$ & Star formation \\
\hline LMC ridge & 0.4 & $>3 \times 10^{21}$ & $>50$ & yes/soon \\
LMC disk & 0.4 & $1-3 \times 10^{21}$ & $\sim 20-50$ & yes, scattered \\
SMC body/wing & 0.2 & $>3 \times 10^{21}$ & $>50$ & yes, throughout \\
SMC tail & 0.1 & $1(-2) \times 10^{21}$ & $\sim 20$ & yes, in pockets \\
Bridge/Stream & $?$ & $<5 \times 10^{20}$ & $\ll 8$ & no \\
\hline
\end{tabular}

The MCs offer a range of gas densities, with the SMC tail being a unique example of a star forming entity close to the critical density, while high-density pockets such as 30 Dor and much of the generally very gas-rich SMC main body support vigorous star formation. The Magellanic Bridge and Stream are apparently too tenuous as they show no

$\dagger$ Although the LMC can be considered a one-armed spiral (see Wilcots, these proceedings), star formation in the LMC is not limited to this particular structure. 


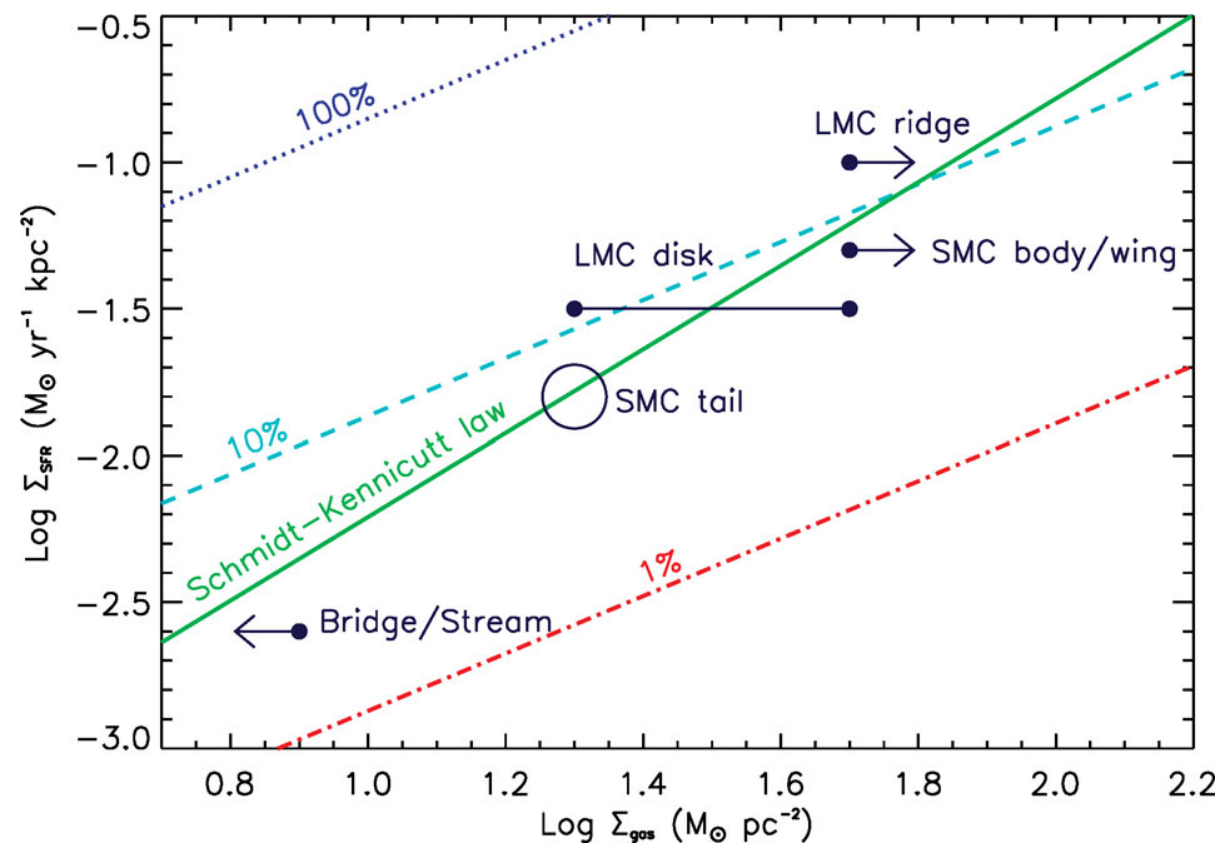

Figure 1. Schmidt-Kennicutt law (Kennicutt 1998) with gas densities for different regions of the Magellanic System indicated (estimated from Nidever et al. 2008, see Table1). Different global star formation efficiencies are also indicated (Kennicutt 1998).

signs of star formation. The transition between quiescent and star-forming ISM within the Magellanic System seems to occur for a gas column density at which Krumholz, McKee \& Tumlinson (2008) predict that molecular clouds become self-shielded. It has now become possible to examine the Schmidt-Kennicutt law on pc scales within individual star-forming clouds in the MCs (see Indebetouw, these proceedings), with the aim of identifying deviations from the global law resulting from differences in the physical processes that work on small scales within galaxies.

The Magellanic Clouds have a low metallicity ISM $\left(0.1-0.4 \mathrm{Z}_{\odot}\right.$, Table 1$)$. This implies that there is in general less dust in the MCs than in the Milky Way, i.e. the gas-to-dust ratio is higher. As we will see in the next section, this might have important implications for the star formation process. The efficiency of some chemo-physical processes are expected to depend on metallicity. Fig. 2 shows typical timescales for several of these processes that are expected to play an important role in the star formation process and their dependence on metallicity (from Banerji et al. 2008). This diagram shows that the relative hierarchy of these processes (i.e. which ones might be more dominant due to shorter timescales) seems to change even in the metallicity range covered by the Magellanic System and the Milky Way. This suggests that metallicity might have an effect on the star formation process, even without having to go back to the conditions prevalent when the very first generations of stars formed.

\section{Star formation and molecular cloud chemistry}

Why is chemistry important for the early stages of the star formation process? First of all chemistry is essential in determining the cooling in a contracting molecular clump. During the onset of gravitational collapse of a dense cloud, sufficiently dense cores can only develop if the heat produced during the contraction can be effectively dissipated. 


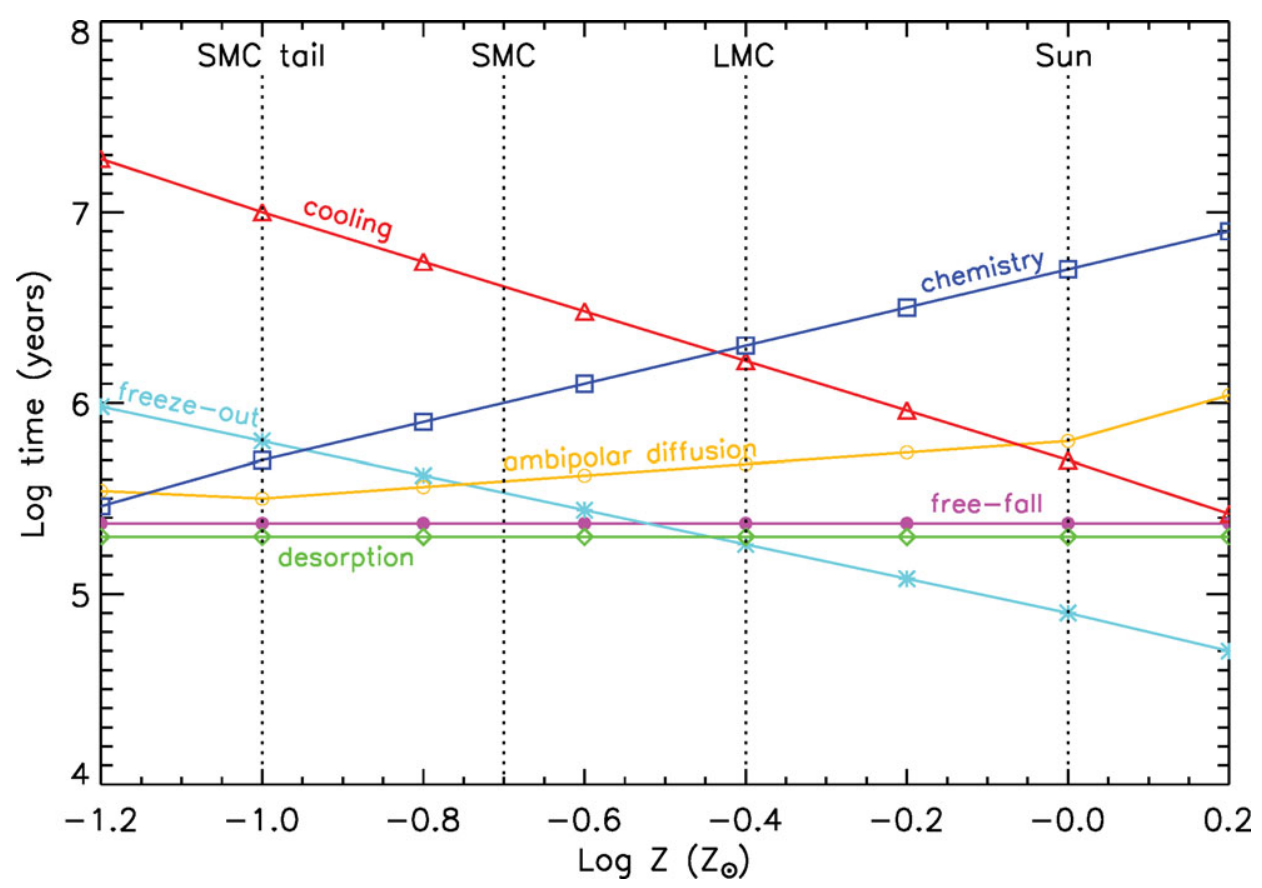

Figure 2. Timescales relevant for the star formation process as a function of metallicity (adapted from Banerji et al. 2008). Processes considered are: free-fall collapse (filled circles), freeze-out (crosses; formation of ice coatings on dust grains), ambipolar diffusion (open circles), desorption (diamonds; process by which molecules formed in ice mantles are returned to the gas phase), cooling (triangles) and ion-molecule chemistry (squares; creation of coolant molecules from atomic gas). Some of these processes and their timescales depend on metallicity. In the metallicity range covered by the MCs and the Galaxy, the relative importance of these processes seems to change.

This cooling occurs mainly via fine-structure lines of $\mathrm{C}$ or $\mathrm{O}$, for instance via the $\mathrm{C}$ II emission line at $158 \mu \mathrm{m}$ and also via rotational transitions of abundant molecules like water. At lower metallicity there are obviously fewer $\mathrm{C}$ and $\mathrm{O}$ atoms and it is expected that this might somehow affect the cooling efficiency (Fig. 2).

Magnetic fields may also play an important role in supporting dense molecular clouds and the cores within them. Magnetic field lines are coupled directly to ions and, via ionneutral collisions, are also coupled to the neutral material. If this latter coupling is weak, then the neutral material decouples from the magnetic field lines and can then respond more freely to any gravitational perturbation (ambipolar diffusion). With fewer metallic atoms available in low metallicity environments, the ionization state of the gas as a whole could be lower and collapse may proceed closer to free fall. On the other hand, less dust shielding and harsher radiation field (see below) might cause metallic atoms to be ionized to a higher degree. Furthermore, it is not clear how important ambipolar diffusion really is (Crutcher, Hakobian \& Troland 2008).

In cold molecular clouds, gas-phase, ice and dust chemistries are strongly inter-linked. Dust grains play an important role by shielding the cold gas in the molecular clouds against ambient UV radiation. Dust grains also provide surfaces onto which chemical reactions occur that would otherwise not be possible. For instance surface chemistry is important in the formation of both $\mathrm{H}_{2}$ and $\mathrm{O}_{2}$ molecules. Ice mantles form on the surfaces of dust grains, leading to depletion of molecules from the gas phase. 
In the MCs the gas-to-dust ratio is higher. If there is less dust then we can expect that the shielding effect of dust opacity is weaker, compounded by the fact that the ambient UV radiation in MCs is harsher than that in our Galaxy (Welty et al. 2006). Chemistry in general might be slower if there is less grain surface available. Extinction curves are different in the diffuse ISM in the MCs (Gordon et al. 2003), possibly due to a different grain size distribution. If dust grains were predominantly smaller in molecular clouds in the MCs, they would provide more surface per unit mass, possibly counteracting the effect of a lower total dust mass. In the denser environment of star forming clouds, grains grow and the rate at which this occurs may be different at low metallicity.

Dust composition is possibly also different. It has been suggested that there might be more carbon-rich dust produced by evolved stars in the SMC (Zijlstra et al. 2006). It is not clear what effect this would have in dust composition as carbon-rich dust is more easily destroyed (cf. van Loon et al. 2008). Nevertheless, if dust composition is different in the MCs, one could expect a different opacity and consequently a different thermal balance. In summary, if the properties of dust grains are intrinsically different in the MCs this could affect chemistry in general and, via cooling, the physics of the star formation process.

\subsection{Ice chemistry}

In cold star forming clouds, ice mantles form on the surface of dust grains. Abundant molecules like water, $\mathrm{CO}_{2}$ (and to some extent $\mathrm{CO}$ ) are largely locked into these ice mantles (Bergin et al. 1995). The most abundant ice species are water (typically $10^{-5}-10^{-4}$ with respect to $\mathrm{H}_{2}$ ), followed by $\mathrm{CO}_{2}$ and $\mathrm{CO}$, with combined abundance $10-30 \%$ with respect to water ice (van Dishoeck 2004). Ice processing also enriches the gas phase: molecules like methanol, formaldehyde and formic acid are possibly formed via UV and cosmic ray processing of ice mantles before being evaporated into the gas phase. Processing also causes segregation of the different ice species and crystallinisation within the ice mantles. Therefore understanding ice chemistry is a powerful route to understand cloud chemistry in general.

Ices have been detected in the envelopes of heavily embedded young stellar objects (YSOs) both in the Milky Way and the MCs. The first evidence of ices in the envelope of a massive YSO in any extra-galactic environment was a serendipitous discovery by van Loon et al. (2005). The Spitzer IRS and ISAAC/VLT spectrum of IRAS 05328-6827 in the LMC shows clear absorption signatures of water ice at $3.1 \mu \mathrm{m}$, methanol ice at 3.5 and $3.9 \mu \mathrm{m}$ and $\mathrm{CO}_{2}$ ice at $15.2 \mu \mathrm{m}$ (Fig. 3). The spectrum also shows a typical silicate dust absorption feature at $10 \mu \mathrm{m}$. Recently Shimonishi et al. (2008) identified a few more embedded YSOs with ice signatures in the LMC. van Loon et al. (2005) suggests that ice processing observed in IRAS 05328-6827 might be an effect of metallicity, as UV radiation is able to deeper penetrate the less dusty envelopes of metal-poor YSOs. This is a tentative result and a more extensive sample, preferably in the SMC, would be needed to constrain any definitive metallicity trend. Three embedded YSOs have been identified in the SMC (van Loon et al. 2008); the ISAAC/VLT spectrum of one of these sources, IRAS 01042-7215, shows clear water ice absorption and hydrogen emission lines, indicating a slightly more evolved object than the LMC example (Fig. 4).

What are the possible effects of low metal content on the chemistry in the envelopes of massive YSOs? Lower carbon and oxygen abundances in the metal-poor ISM of the MCs lead to lower gas-phase CO abundances (Leroy et al. 2007). One could therefore also expect a lower $\mathrm{CO}$ and $\mathrm{CO}_{2}$ content of the ice mantles that coat the dust grains. On the other hand a lower dust fraction leads to reduced shielding from an already harder interstellar radiation field. A high degree of ice processing could thus be expected as 


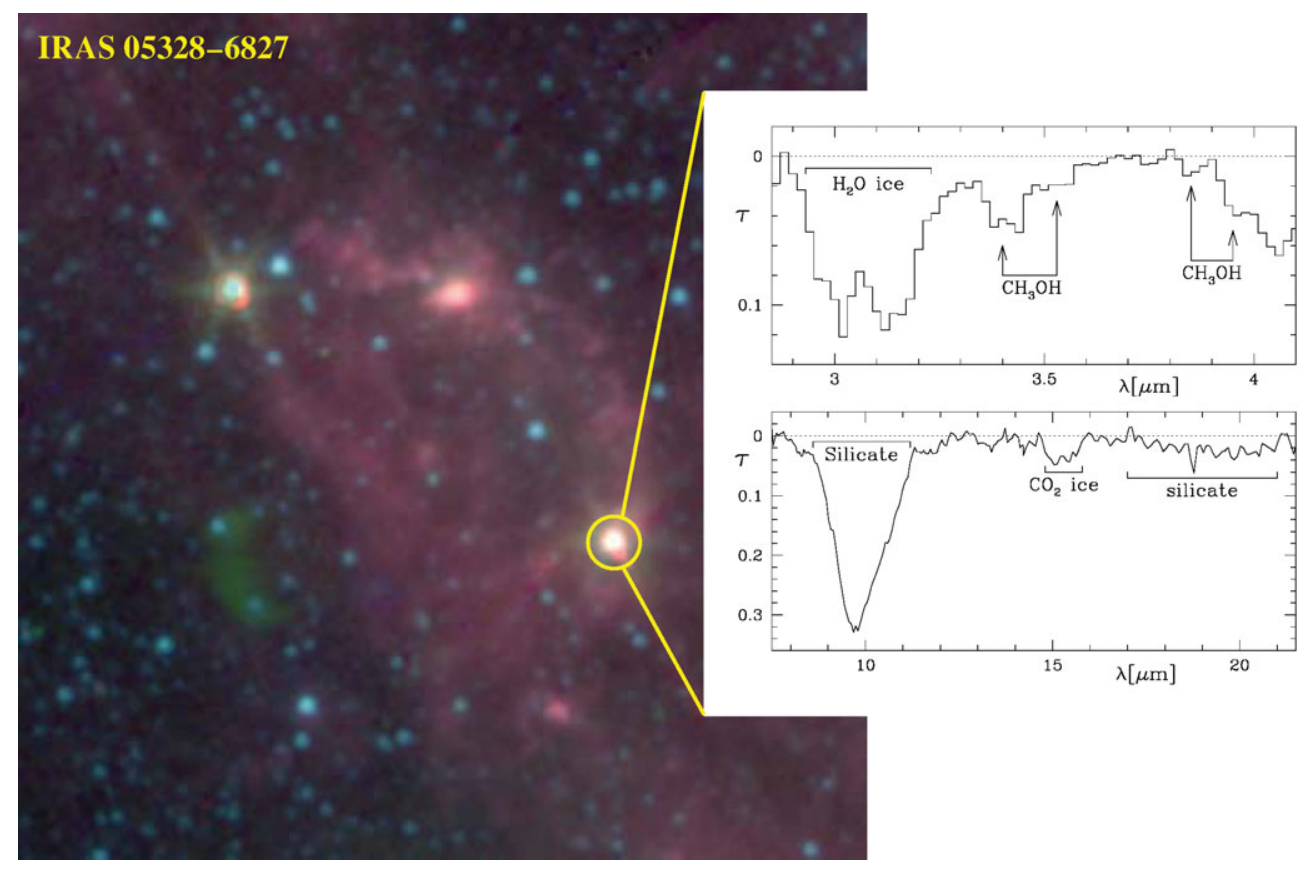

Figure 3. IRAS 05328-6827 in the LMC, the first extra-galactic embedded YSO spectroscopically identified by van Loon et al. (2005). The image is a 3-colour IRAC image; the spectra clearly show absorption features due to water and $\mathrm{CO}_{2}$ ices as well as silicate dust.

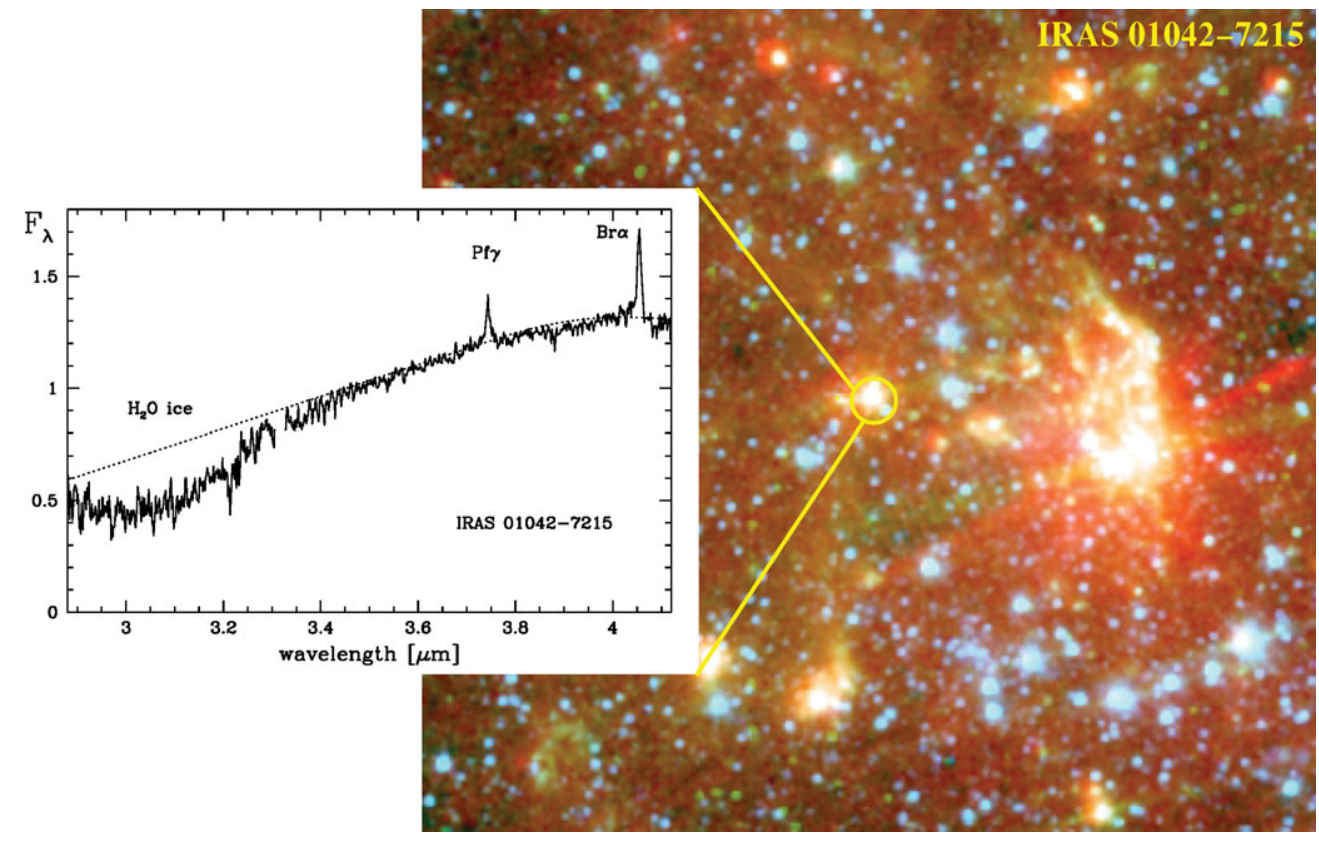

Figure 4. IRAS 01042-7215 in the SMC, a YSO identified in the SMC (van Loon et al. 2008). The image is a 3-colour IRAC image; the spectrum clearly show absorption features due to water ice, as well as emission lines, indicating a more evolved object than IRAS 05328-6827 (Fig. 3). 
well as a higher degree of crystallinity and ice species segregation. While the first effect could be expected to depress the abundances of all species when compared to Galactic embedded YSOs, the second effect would enhance more complex ice species mainly at the expense of water ice. It is not clear at present which, if any, of these effects dominates. By comparing YSO samples in the SMC, LMC and the Milky Way we can probe metallicities from $0.1-1 \mathrm{Z}_{\odot}$, allowing us to assess the effect of metallicity on ice chemistry.

Firstly, we will have to construct reliable samples of YSOs in the MCs, selected in a more systematic way, to be compared with Galactic samples. The Spitzer Space Telescope imaging surveys of the MCs - SAGE (Meixner et al. 2006), $\mathrm{S}^{3} \mathrm{MC}$ (Bolatto et al. 2007) and SMC-SAGE (Gordon et al., in preparation) - have identified large numbers of YSO candidates (Whitney et al. 2008). Follow-up spectroscopic surveys (including SAGE-SPEC and SMC-IRS) target a sizeable YSO sample, that will allow the study of ice features in the mid-IR range. AKARI is also being used to investigate ice features on many such objects (Shimonishi et al. 2008) in the near-IR, as are groundbased investigations (Oliveira et al., in preparation).

Observations of Galactic YSOs show that the bands of the main ice species are better fitted by laboratory profiles that include admixtures of other ices (Pontoppidan et al. 2008). Furthermore, from the previous paragraph it is clear that relative ice abundances, not individual ice species, hold the key to isolate any environmental chemistry effect. Another important point to take into account is that in the MCs we can only expect to study the most massive embedded YSOs, thus one needs to carefully consider the objects' luminosity before comparing the samples. The analysis of the spectral energy distributions of such objects allows to constrain not only the objects' luminosity and evolutionary stage but also dust properties that together with ice chemistry will build a coherent picture of the early star formation process.

One of the major advances since the previous MC Symposium has been the availability of facilities like Spitzer which now allow us to study in detail the properties of embedded YSOs outside our Galaxy. We can thus sample star formation environments that are significantly different from those encountered in the Milky Way, with the real possibility of understanding how metallicity influences the early stages of star formation. At the same time, we can now also study global properties of the resulting young stellar populations and investigate for instance how the Initial Mass Function (IMF) reflects their parent environment.

\section{From cloud collapse to the stellar IMF}

The stellar IMF is determined by a range of inter-linked physical processes: cooling, turbulence, fragmentation, feedback, rotation, magnetic fields etc. The IMF is a rather simplistic snapshot of a very complicated process, and we still struggle to fully understand it and explain its main properties.

At the extreme low metallicities of the early Universe, the IMF is believed to have been top heavy, i.e star formation events gave rise only to extremely massive stars, due mainly to the inability of primordial gas to efficiently cool at low temperatures (Abel, Bryan \& Norman 2002). As these populations die and new ones emerge, the Universe gets progressively enriched with copious amount of metals. Once metallicities of the order of $10^{-3}$ to $10^{-5} \mathrm{Z}_{\odot}$ are reached, enough metals are present to allow for more efficient cooling, through fine-structure and molecular transitions, as well as continuum emission from dust produced in supernova explosions. This more efficient cooling allows the gas that will form the next generation of stars to reach lower temperatures, and therefore smaller clumps can be created via fragmentation (e.g., Smith \& Sigurdsson 2007). That is to say 
lower-mass stars can form and the peak of the IMF shifts to lower masses. Thus, once the metal content in the Universe reaches this metallicity threshold stellar populations are characterised by essentially a Salpeter-like IMF with a turn-over or characteristic mass at lower masses, as observed in the solar neighbourhood (Kroupa 2001; Chabrier 2005).

The present day local IMF seems to be universal, but significant variations are observed mainly in more extreme environments, like regions that formed at high redshift (Elmegreen 2008). The IMF can be defined as $\xi(\log M) \propto M^{\Gamma}$, where $\Gamma$ is the IMF slope. Observationally, the IMF is found to have a slope of $\Gamma \sim-1.35$ (commonly referred to as the "Salpeter slope", Salpeter 1955) down to below a solar mass; it then flattens out somewhat (the so-called IMF plateau) and reaches a peak at about $0.3 \mathrm{M}_{\odot}$, falling sharply into the brown dwarf regime. Theoretical considerations actually struggle to explain the observed IMF constancy in a wide variety of environments (Kroupa 2008). However, this refers mostly to the slope of the IMF at higher masses or to integrated stellar populations; only very recently are we able to probe directly the low-mass IMF in environments that may be distinct to those prevalent in the Milky Way.

In what way could we expect metallicity to influence the lower mass IMF? Based on numerical simulations it has been proposed that the characteristic mass scales with the thermal Jeans mass at the onset of collapse (Bate \& Bonnell 2005). One could then intuitively expect the IMF properties to vary with the environmental conditions, via for instance the dependence of the Jeans mass on the temperature and metallicity in the molecular core. However, Elmegreen et al. (2008) show that, if grain-gas coupling is taken into account, the thermal Jeans mass should depend only weakly on environmental factors like density, temperature, metallicity and radiation field. In particular, the dependence of the Jeans mass with metallicity could be only as $Z^{-0.3}$. It is also possible that the characteristic mass is not linked to the Jeans mass, depending instead on core subfragmentation and protostellar feedback (Elmegreen 2008).

What have we learned so far from constraining the IMF in the MCs? One of the first regions investigated to characterise the high-mass IMF outside our Galaxy was R 136 in the LMC. In the early days, it was thought that R 136 was a single super-massive star (Cassinelli, Mathis \& Savage 1981), suggesting an IMF with different properties to those observed in the Galaxy. Modern instrumentation however quickly resolved R 136 into a massive star cluster (Weigelt \& Baier 1985). In fact, the most massive stars observed in the MCs have masses of the order of $\sim 150 \mathrm{M}_{\odot}$ (Massey \& Hunter 1998), consistent with Galactic massive objects. We should point out that these are observed masses. As discussed in Elmegreen (2008), very massive stars loose a substantial amount of mass extremely quickly. Thus this observed maximum mass is not necessarily the maximum mass as far as the star formation process is concerned.

The IMF of several associations in the MCs is found to have a Salpeter slope down to about $1-2 \mathrm{M}_{\odot}$ (Sirianni et al. 2000; Selman \& Melnick 2005; Kumar, Sagar \& Melnick 2008, to name but a few). To study the lower-mass IMF, we need to investigate populations younger than about $10 \mathrm{Myr}$, as these are less affected by dynamic evolution effects. Young solar-mass stars were firstly identified using near-IR images or $\mathrm{H} \alpha$ emission, for instance in 30 Doradus (Brandl et al. 1996) and near SN 1987 A (Panagia et al. 2000). More recently a number of Hubble Space Telescope (HST) surveys have allowed the detection of large numbers of pre-main-sequence (PMS) objects in H II regions in the Clouds, down to below half a solar mass (e.g. Nota et al. 2006; Gouliermis et al. 2006, 2007).

Two young star forming regions in the SMC have been recently under very close scrutiny (see also contributions by Gouliermis and Sabbi in this volume). NGC 602 in the wing of the SMC shows that star formation can be triggered in low-density environments, 
probably by compression and turbulence associated with H I shell interactions (Nigra et al. 2008). The massive cluster in the center has many PMS stars associated with it (Gouliermis, Quanz \& Henning 2007) and embedded ongoing star formation has been detected towards the rim of the H II region (Carlsson et al. 2007). NGC 346 on the other hand is the largest $\mathrm{OB}$ association in the SMC and it has a more complex gas and dust morphology than NGC 602. A rich PMS has been discovered in this region (Nota et al. 2006) and star formation is still ongoing in its denser parts (Simon et al. 2007). It has been proposed that several star formation episodes have taken place in NGC 346 (Sabbi et al. 2007; Hennekemper et al. 2008). For both these young regions the IMF is found to be Salpeter-like down to about a solar mass (Schmalzl et al. 2008; Sabbi et al. 2008); below that mass incompleteness becomes a serious issue.

Even with HST imaging we are unable to fully sample the IMF plateau and determine the characteristic mass of young stellar populations in the MCs. But even when new instrumentation allows us to do so, we might struggle to find conclusive answers. If the characteristic or peak mass depends on metallicity as proposed by Elmegreen et al. (2008), in the MCs we could expect it to be about $0.4-0.6 \mathrm{M}_{\odot}$, where in the Galaxy it typically is $0.3 \mathrm{M}_{\odot}$. Furthermore, even in the Milky Way we observe variations in the peak mass that seem to be significant (Luhman et al. 2007; Oliveira et al. 2009) however we are as yet unable to explain these differences. Another possible complication is that the peak mass also depends (albeit weakly) on the density and radiation field, and in the MCs we do sample lower densities and harsher radiation fields. Therefore a metallicity effect on the IMF properties might prove difficult to isolate.

\section{Feedback and triggering}

Another factor we have to take into account is that star formation has a cyclic component to it: feedback from massive stars can quench/prolong a star formation episode and maybe even trigger new such events. If feedback effects are different at low metallicity, can this affect the outcome of a star formation event?

Infant massive stars start to shape their environments very early in their evolution, by ionizing the gas and heating the dust (Oey \& Clarke 2007). Stellar UV radiation and winds create expanding blisters of ionized gas. At the interfaces between these H II regions and the cold molecular cloud, the heating powers shocks that compress the molecular material. Supernova explosions also disrupt severely the neighbouring ISM. In the first instance, massive stars violently destroy their parent molecular clouds. However, observational evidence indicates that at the rims of giant $\mathrm{H}$ II regions star formation is ongoing, NGC 6611 and the Eagle Nebula in the Milky Way being one such example (Oliveira 2008). This seems to suggest that under the right conditions, radiative and/or mechanical feedback of massive stars can trigger further star formation in the remnant molecular gas (see review by Chu in this volume).

The observed properties of massive stars in the Magellanic Clouds show some important differences when compared to their galactic counterparts (see review by Evans in this volume). Massive stellar winds are slower and less dense in the low metallicity environment of the MCs (Mokiem et al. 2007). For the same stellar mass, massive stars in the Clouds are also hotter than their Galactic counterparts (Massey et al. 2005), which could compensate slightly the metallicity effect on the wind as hotter stars drive faster outflows. The lower dust-to-gas ratio in the MCs implies that dust shielding is less efficient and radiative feedback from massive stars may thus be more effective at lower metallicity. All this seems to suggest that massive star feedback might operate differently at low metallicity. But do we know how this affects the triggering of star formation? 

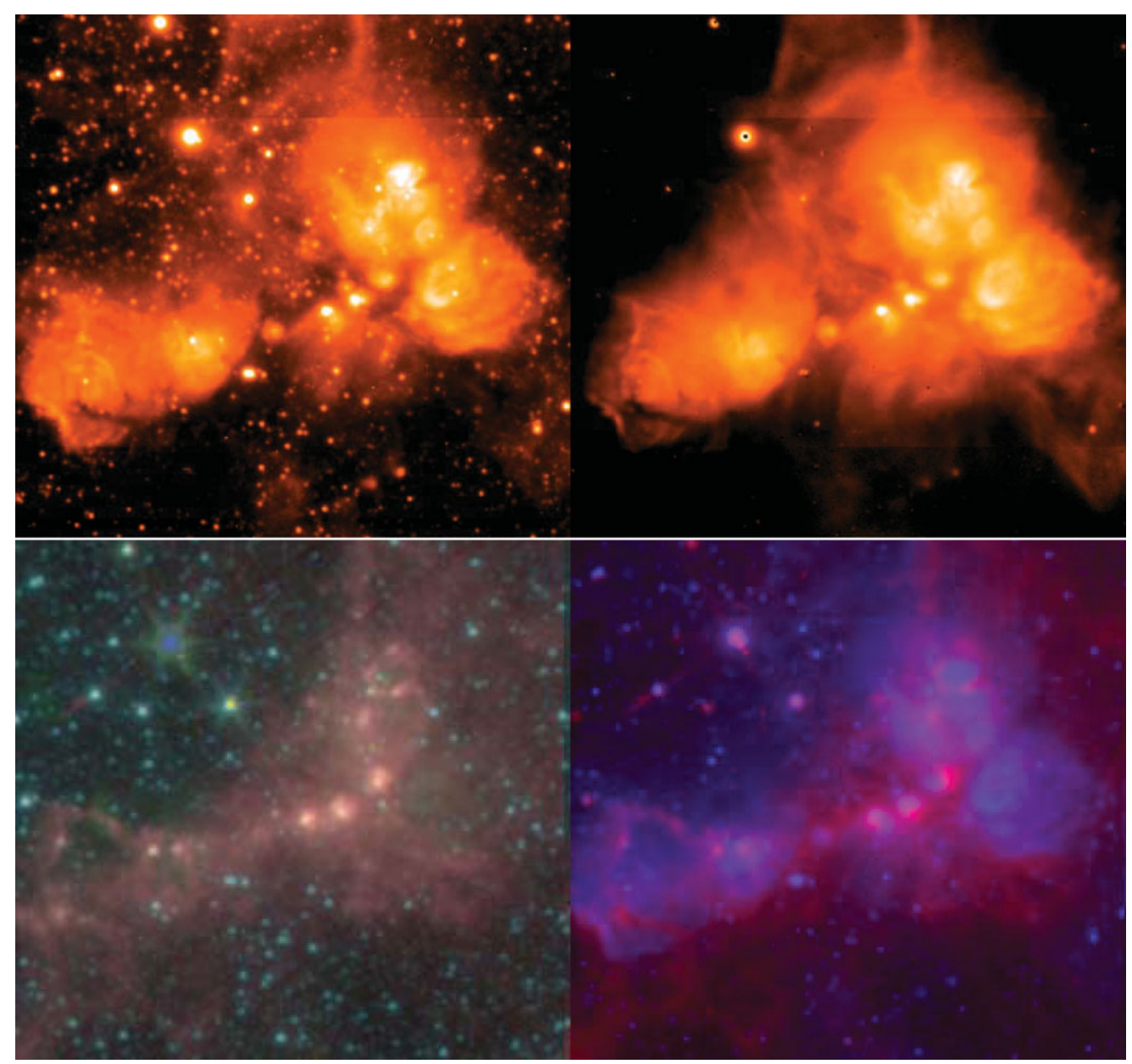

Figure 5. Multi-wavelength images of N 113 in the LMC (Oliveira et al. 2006). The images at the top are the $\mathrm{H} \alpha+$ continuum (left) and $\mathrm{H} \alpha$ emission (right), showing the complex structure of the hot gas. The image on the bottom left shows an IRAC/Spitzer 3-colour composite, showing the location of the dense dust lane in the center of the region. The image on the bottom right shows a composite of $\mathrm{H} \alpha+$ continuum with IRAC/Spitzer $8 \mu \mathrm{m}$ dust emission. It shows to dramatic effect how the bubbles of ionized gas created by the massive stars are compressing the dense lanes of dust. Star formation is ongoing in the compressed dust, as shown by the presence of maser emission (Oliveira et al. 2006).

N 113 is a small H II region in the LMC. Multiple young stellar populations are associated with this region (Bica, Claria \& Dottori 1992) and star formation is ongoing within radio continuum sources in the central part of the nebula (Wong et al. 2006), as pinpointed by the detection of water and $\mathrm{OH}$ maser emission (Lazendic et al. 2002; Brooks \& Whiteoak 1997). Fig. 5 shows in detail the interplay between the ionized gas and the molecular material in N 113. Oliveira et al. (2006) use $\mathrm{H} \alpha$ emission and continuum images together with IRAC/Spitzer images to show that the ionized gas bubbles created by the massive stars in the region are compressing the dense lanes of molecular material where star formation is ongoing. Further persuasive evidence for triggered star formation in the MCs is found at the rims and interfaces of LMC supergiant shells where ongoing star formation is occurring (see contribution by $\mathrm{Chu}$, this volume). This paints 
the picture that star formation triggered by massive stars and their interaction with the ISM also occurs in the Clouds.

One problem with analysing the processes that can trigger star formation is to establish a cause-effect relationship: it is very difficult to distinguish between sequential star formation that occurs without the need for extra triggers and star formation that occurs only due to the direct action of the massive stars. One of the few examples where triggering may have been proved is the superbubble N51D in the LMC. By measuring the thermal pressure in a dust globule and comparing it to ambient conditions, Chu et al. (2005) find that star formation in the globule may have been induced recently by the thermal pressure in the superbubble interior.

As we have seen in Section 2, in the MCs there are other mechanisms that could trigger star formation: ram pressure and/or tidal effects resulting from interactions within the Magellanic System and with the Milky Way. Even though we know that massive stars have different observed properties at low metallicity, it is not clear whether this significantly influences star formation triggering.

\section{Final remarks}

I reviewed what we know about the star formation environment in the Magellanic Clouds, in particular concerning gas density and metallicity. I described how particular components of the star formation process, namely, the chemistry of the early embedded stages, stellar IMF and stellar feedback and triggering, might operate differently in environments with low metal abundance as in the Magellanic Clouds. The Magellanic System offers an ideal laboratory to study both the details and global properties of star formation at low metallicity. This kind of studies has only recently become possible; with so much new data and exciting facilities coming on, we have very much to learn!

\section{References}

Abel, T., Bryan, G. L., \& Norman, M. L. 2002, Sci, 295, 93

Banerji, M., Viti, S., Williams, D. A., \& Rawlings, J. M. C. 2008, ApJ, in press, arXiv0810.3662 Bate, M. R. \& Bonnell, I. A. 2005, MNRAS, 356, 1201

Bekki, K. \& Chiba M. 2007, PASJ, 24, 21

Bergin, E. A., Langer, W. D., \& Goldsmith, P. F. 1995, ApJ, 441, 222

Bica, E. L., Claria, J. J., \& Dottori, H. 2002, AJ, 103, 1859

Bolatto, A. D., Simon, J. D., Stanimirović, S., et al. 2007, ApJ, 655, 212

Brandl, B., Sams, B. J., Bertoldi, F., et al. 1996, ApJ, 466, 254

Brooks, K. \& Whiteoak, J. B. 1997, MNRAS, 291, 395

Carlson, L. R., Sabbi, E., Sirianni, M., et al. 2007, ApJ, 665, 109

Cassinelli, J. P., Mathis, J. S., \& Savage, B. D. 1981, Sci, 212, 1497

Chabrier G. 2005, in E. Corbelli, F. Palla, \& H. Zinnecker (eds.), The Initial Mass Function 50 years later, ApSS Library 327 (Dordrecht: Springer), p. 41

Chu, Y.-H., Gruendl, R. A., Chen, C.-H. R., et al. 2005, ApJ, 634, 189

Crutcher, R. M., Hakobian, N., \& Troland, T. H. 2008, in press, arXiv0807.2862

de Boer, K. S., Braun, J. M., Vallenari, A., \& Mebold, U. 1998, A\& A, 329, L49

Elmegreen, B. G. 2009, in The Galaxy Disk in Cosmological Context, IAU Symposium 254, arXiv0810.5406

Elmegreen, B. G. 2008, in The Evolving ISM in the Milky Way and Nearby Galaxies: Recycling in the Nearby Universe, $4^{\text {th }}$ Spitzer Science Center Conference, arXiv:0803.3154

Elmegreen, B. G., Klessen, R. S., \& Wilson, C. D. 2008, ApJ, 681, 365

Gordon, K. D., Clayton, G. C., Misselt, K. A., Landolt, A. U., \& Wolff, M. J. 2003, ApJ, 594, 279

Gouliermis, D. A., Brandner, W., \& Henning, T. 2006, ApJ, 636, 33 
Gouliermis, D. A., Henning, T., Brandner, W., Dolphin, A. E., Rosa, M., \& Brandl, B. 2007, ApJ, 665, 27

Gouliermis, D. A., Quanz, S. P., \& Henning, T. 2007, ApJ, 665, 306

Harris, J. 2007, ApJ, 658, 345

Hennekemper, E., Gouliermis, D. A., Henning, T., Brandner, W., \& Dolphin, A. E. 2008, ApJ, 672,914

Kennicutt, R. C., Jr. 1998, ApJ, 498, 541

Kroupa, P. 2001, MNRAS, 322, 231

Kroupa, P. 2008, in G. Israelian \& G. Meynet (eds.), The Metal Rich Universe (Cambridge, UK: Cambridge University Press), p. 227

Krumholz, M. R., McKee, C. F., \& Tumlinson, J. 2008, ApJ in press, arXiv:0811.0004

Kumar, B., Sagar, R., \& Melnick, J. 2008, MNRAS, 386, 1380

Lazendic, J. S., Whiteoak, J. B., Flamer, I., Harbinson, P. D., \& Kuiper, T. B. H. 2002, MNRAS, 331,969

Leroy, A., Bolatto, A. D., Stanimirović, S., Mizuno, N., Israel, F., \& Bot, C. 2007, ApJ, 658, 1027

Luhman, K. L., Joergens, V., Lada, C., Muzerolle, J., Pascucci, I., \& White, R., 2007, in B. Reipurth, D. Jewitt, and K. Keil (eds.), Protostars and Planets V, (Tucson: University of Arizona Press), p. 443

Massey, P. \& Hunter, D. A. 1998, ApJ, 493, 180

Massey, P., Puls, J., Pauldrach, A. W. A., Bresolin, F., Kudritzki, R. P., \& Simon, T. 2005, ApJ, 627, 477

Meixner, M. Gordon, K. D., Indebetouw, R. et al. 2006, AJ, 132, 2268

Mokiem, M. R., de Koter, A., Vink, J. S., et al. 2007, A\&A, 473, 603

Nidever, D. L., Majewski, S. R., \& Burton W. B. 2008, ApJ, 679, 432

Nigra, L., Gallagher, J. S., III, Smith, L. J., Stanimirović, S., Nota, A., \& Sabbi, E. 2008, PASP, 120,972

Nota, A., Sirianni, M., Sabbi, E., et al. 2006, ApJ, 640, 29

Oey, M. S. \& Clarke, C. J. 2007, in M. Livio \& E. Villaver (eds.), Massive Stars: From Pop III and GRBs to the Milky Way, (Cambridge, UK: Cambridge University Press), astro-ph/0703036

Oliveira, J. M., van Loon, J.Th., Stanimirović, S., \& Zijlstra, A.A. 2006, MNRAS, 372, 1509

Oliveira, J. M., Jeffries R. D., \& van Loon J. Th. 2008, 2009, MNRAS, 392, 1034

Oliveira, J. M. 2008, in B. Reipurth (ed.), The Handbook of Star Forming regions, in press, arXiv:0809.3735

Panagia, N., Romaniello, M., Scuderi, S., \& Kirshner, R. P. 2000, ApJ, 539, 197

Pontoppidan, K. M., Boogert, A. C. A., Fraser, H. J., et al. 2008, ApJ, 678, 1005

Sabbi, E., Sirianni, M., Nota, A., et al. 2008, AJ, 135, 173

Sabbi, E., Sirianni, M., Nota, A., et al. 2007, AJ, 133, 44

Salpeter, E. E. 1955, ApJ, 121, 161

Schmalzl, M., Gouliermis, D. A., Dolphin, A. E., \& Henning, T. 2008, ApJ, 68, 290

Schmidt, M. 1959, ApJ, 129, 243

Selman, F. J. \& Melnick, J. 2005, A\&A, 443, 851

Shimonishi, T., Onaka, T., \& Kato, D., et al. 2008, ApJ, 686, L99

Simon, J. D., Bolatto, A, D., \& Whitney, B. A., et al., 2007, ApJ, 669, 327

Sirianni, M., Nota, A., Leitherer, C., De Marchi, G., \& Clampin, M. 2000, ApJ, 533, 203

Smith, B. D. \& Sigurdsson, S. 2007, ApJ, 661, L5

van Dishoeck, E. 2004, ARAA, 42, 119

van Loon, J. Th., Oliveira, J. M., Wood , P. R., et al. 2005, MNRAS, 364, L71

van Loon, J. Th., Cohen, M., Oliveira, J. M., et al. 2008, A $₫ A, 487,1055$

Weigelt, G. \& Baier, G. 1985, A\& A, 150, 18

Welty, D. E., Federman, S. R., Gredel, R., Thorburn, J. A., \& Lambert, D. L. 2006, ApJS, 165, 138

Whitney, B. A., Sewilo, M., Indebetouw, R., et al. 2008, AJ, 136, 18

Wong, T., Whiteoak, J. B., Ott, J., Chin, Y.-N., \& Cunningham, M. R. 2006, ApJ, 649, 224

Zijlstra, A. A., Matsuura M., Wood P. R., et al. 2006, MNRAS, 370, 1961 